Key words: Magnetic Resonance Spectroscopy, Traumatic Brain Injury, Depression.

\title{
Lesion Location in Depression Post Traumatic Brain Injury Using Magnetic Resonance Spectroscopy: Preliminary Results from a Pilot Study
}

\author{
Vani Rao, MD* \\ Jennifer Spiro, MS* \\ Mahaveer Degoankar, $\mathrm{PhD}^{\star *}$ \\ Alena Horská, $\mathrm{PhD}^{* *}$ \\ Paul B. Rosenberg, MD* \\ David M. Yousem, MD** \\ Peter B. Barker ${ }^{\star *}$ \\ D. Phil
}

* Division of Geriatric Psychiatry \& Neuropsychiatry, Department of Psychiatry, Johns Hopkins School of Medicine.

Baltimore, MD 21287

** Division of Neuroradiology, Russell H Morgan Department of Radiology and Radiological Science, Johns Hopkins School of Medicine. Baltimore, MD 21287

USA

\footnotetext{
ABSTRACT - Objective: To determine the metabolic status of the brain in post traumatic brain injury(TBI) depression using proton magnetic resonance spectroscopy (MRS).

Design: Case-control study including 5 TBI depressed subjects and 5 age matched non-TBI non-depressed controls.

Methods: Metabolic status was assessed using proton MRS. Ratios of N-acetylaspartate (NAA), choline (Cho) and total creatine $(\mathrm{Cr})$ were calculated in frontal cortex, basal ganglia and thalamus.

Results: NAA/Cho or NAA/Cr ratios were significantly reduced in the TBI depressed group compared to controls in frontal cortex, basal ganglia and thalamus.

Conclusion: Reduced levels of NAA in frontal regions, basal ganglia and thalamus in TBI depression suggest neuronal damage or dysfunction which may be a associated with the primary brain injury or with depressed mood.
} 


\section{Introduction}

Traumatic brain injury (TBI) is a public health problem of increasing importance in the U.S. TBI survivors often suffer multiple neuropsychiatric sequelae (McAllister 1992) including depressed mood, with estimates of the prevalence of depression ranging from $13 \%$ at 1 year after trauma (Deb et al. 1999) to $60 \%$ at 8 years (Hibbard et al. 1998). Depressed mood can contribute to disability after TBI by interfering with rehabilitation and exacerbating cognitive deficits, as well as contributing directly to suffering and morbidity (Jorge et al. 1994).

Post TBI depression can be divided into early onset / transient depression, which occurs within 1 to 3 months after injury and lasts for a few days to weeks, and late onset / persistent depression, which can occur several months after the injury and may last for months afterwards (Jorge et al. 1993). Jorge et al. $(1993 ; 2004)$ examined the anatomical basis of post-TBI depression with computerized tomography (CT) and magnetic resonance imaging (MRI). Early onset depression is associated with lesions in the left dorsolateral frontal and/or left basal ganglia regions, but this association is lost 3 months after injury. These results suggest that earlyonset transient depression is associated with disrupted brain physiology associated with the injury, whereas late-onset depression may be a psychological reaction to physical and cognitive impairment.

Proton magnetic resonance spectroscopy (MRS) and magnetic resonance spectroscopic imaging (MRSI) are noninvasive in vivo techniques to assess the metabolic status of the brain which can quantify selected neurochemical moieties, including $\mathrm{N}$-acetylaspartate (NAA), creatine and phosphocreatine $(\mathrm{Cr})$, choline-containing compounds
(Cho), lactate, myo-inositol, and glutamate and glutamine (Brooks et al. 2001). NAA levels have been validated as a marker of neuronal and axonal integrity and function (Bachelard \& Badar-Goffer 1993). NAA is reduced following TBI, reflecting diffuse axonal injury or metabolic depression (Brooks et al. 2001). Elevation of Cho signal indicates cell membrane breakdown or cell proliferation or both (Brooks et al. 2001). Creatine and phosphocreatine are involved in energy metabolism and the $\mathrm{Cr}$ signal is decreased in conditions associated with cell death. Standard analyses of MR spectra include quantifying brain levels of NAA, Cho, and $\mathrm{Cr}$, as well as calculating metabolite ratios. Preliminary MRS studies in mood and anxiety disorders have reported alterations in brain levels of Cho, myo-inositol, NAA, and gamma-aminobutyric acid (Murata et al. 2001, Kumar et al. 2002, Moore et al. 2002). To date there are no published results of MRS in post-TBI depression.

We present a pilot study using MRS to compare brain NAA, Cho, and Cr levels in subjects with post-TBI depression to control subjects lacking depression or history of TBI. We hypothesized that TBI depressed subjects would have decreased NAA/Cho and/or NAA/Cr ratios in the frontal gray matter (GM) and basal ganglia compared to controls. The frontal cortex and basal ganglia have been implicated in structural imaging studies of the pathogenesis of postTBI depression (Jorge 2004).

\section{Methods}

All TBI depressed subjects underwent a complete neuropsychiatric evaluation and brain imaging scans. TBI depressed subjects were recruited by advertisements in local 
newspapers, health magazines and head injury support groups. Inclusion criteria included age 18 years or older, date of TBI between 3 and 24 months of MRS study, no history of diagnosable mood disorder prior to TBI, Mini Mental State Examination (MMSE) $>24$, stable medical history prior to injury, sufficient cognitive capacity to provide consent. Depressed subjects were required to meet DSM-IV criteria for Major Depressive Episode, and to have met these criteria only in the period after TBI. Exclusion criteria included open head injury, severe unstable medical diseases (cardiac, renal, hepatic, pulmonary), repeated $(>2)$ head injury, stroke or encephalitis, alcohol or other illicit drugs use in past 30 days, claustrophobia, pacemakers, defibrillator wires, cochlear implants, metal implants, ferromagnetic surgical clips in the brain and/or history of metal in the eyes, and pregnancy. Control subjects without history of TBI who never met the DSM-IV criteria for major depressive episode were recruited from the community. They were age-matched (+/- 7 years) to the TBI depressed subjects. Subjects were similar in age $(\mathrm{t}[8]=-.32, \mathrm{p}=.76)$.

\section{MRSI Methodology}

MRI examinations were performed on a 1.5 Tesla Gyroscan ACS NT scanner (Philips Medical Systems, Best, The Netherlands).

Proton MRSI was performed using a multi-slice spin-echo (SE) sequence with outer volume suppression (Duyn et al. 1993). Three oblique axial slices were acquired with a $15 \mathrm{~mm}$ thickness and a gap of $2.5 \mathrm{~mm}(\mathrm{TR} / \mathrm{TE}=2000 / 280 \mathrm{~ms}$, acquisition matrix $28 \times 28 \times 256, \mathrm{FOV}=24 \mathrm{~cm})$. The echo signal was digitized with 256 data points and a spectral width was $1,000 \mathrm{~Hz}$.
Water suppression was accomplished with a single Chemical Shift Selective (CHESS) pulse with a bandwidth of $110 \mathrm{~Hz}$.

T1-weighted SE MR images $(\mathrm{TR} / \mathrm{TE}=$ $400 / 20 \mathrm{~ms}, 15 \mathrm{~mm}$ slice thickness) were recorded at the same slice locations as the MRSI data set for anatomical correlation.

The in-house software "csx" was used to process the MRSI data sets. Multi-slice twodimensional MRSI data sets were processed by three-dimensional Fourier transformation with cosine filters in the spatial (phaseencoding) domains and exponential line broadening of $3 \mathrm{~Hz}$, zero-filling to 8.192 data points, and a high-pass convolution filter to remove the residual water signal (50 $\mathrm{Hz}$ stop-band) in the time-domain. A susceptibility correction (i.e. left- or right shifting of each spectrum in the frequency domain on a voxel by voxel basis) was applied after magnitude calculation, based on the chemical shift of NAA of $2.02 \mathrm{ppm}$. Signals of total NAA, Cho, and Cr were fitted to a Guassian lineshape using a simplex routine (Soher et al.1996).

Metabolite ratios of NAA/Cho, NAA/Cr and $\mathrm{Cho} / \mathrm{Cr}$ were calculated from several regions of interest: frontal white matter, frontal gray matter, basal ganglia and thalamus, from both hemispheres. Care was taken to select a voxel entirely encompassed in the particular region of interest to avoid contamination from other tissue type or cerebrospinal fluid. Corresponding regions were selected in the case and control groups. However, corrections for voxel tissue composition was not done.

An attempt was made to evaluate 2 spectra from each region and the average values of metabolite ratios were used for the analyses. In some cases only 1 spectrum was evaluated due to low data quality of the second spectrum. 
The following anatomical criteria was used to select different regions: (a) Frontal gray mater: Lateral aspect of the superior/middle frontal gyrus (b) Frontal white matter: Frontal radiation of the corpus callosum (c) Basal Ganglia: Lateral portion of the elongated cone-shaped gray matter structure laterodoral to the caudate nucleus and medial to the insula and (d) Thalamus: Medial gray/white matter structure on the lateral wall of the 3rd ventricle. Care was taken to avoid regions with visible lesions on magnetic resonance imaging (MRI), as the aim of our study was to assess biochemical changes in areas not associated with visible MRI lesions.

Figure 1 shows the MRSI voxel locations. Figure 2 shows the areas of interest from which data were collected for the analysis.

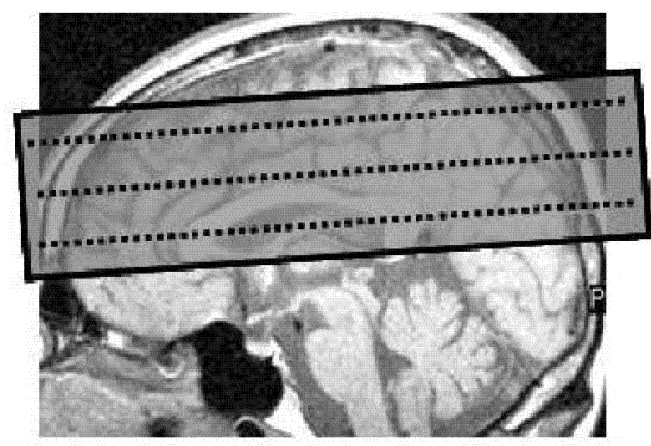

Figure 1. A saggital MR localizer image showing the location of the three MRSI slices, parallel to the anterior commissure-posterior commissure line.
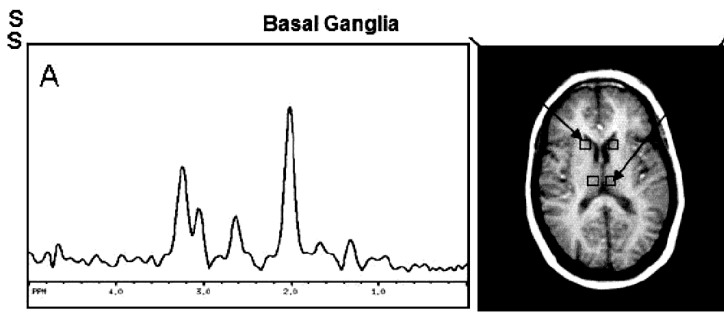

Thalamus
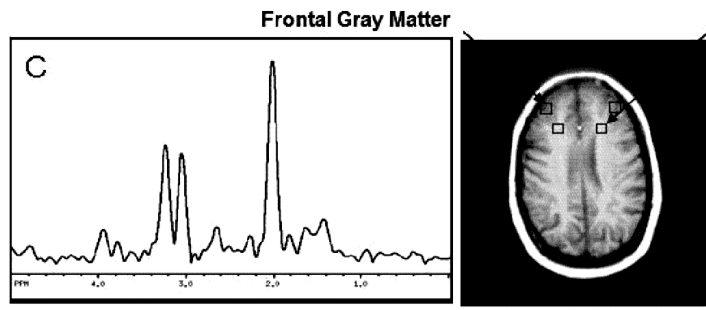

Frontal White Matter

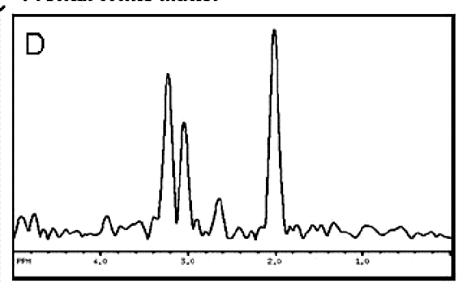

Figure 2. Representative MR Spectra from four regions of interest: A. Basal Ganglia; B. Thalamus; C. Frontal Gray Matter; D. Frontal White Matter, in Brain. Localizer T1 weighted images show 2nd \& 3 rd slice from were the four ROIs were selected. 


\section{Statistical Analysis}

Independent $\mathrm{t}$ tests were used to determine the difference in these ratios between TBI depressed subjects and controls. Pvalue was set at 0.05 . Effect size was calculated by taking the mean difference in ratios (control-case) divided by the sample standard deviation for each variable.

Because this is a preliminary study with a small sample size $(n=10)$, it was not practical to use a Bonferroni correction or other adjustment for multiple comparisons in the analyses.

\section{Results}

Demographics and baseline clinical variables are presented in Table I. When the 5 depressed TBI subjects were compared to 5 normal controls, the NAA/Cho or NAA/Cr ratios were significantly decreased in the TBI depressed group compared to normal controls in the frontal GM, basal ganglia, and the thalamus. We also had moderate to large effect size (see Table II). No difference between the two groups was noted in the frontal white matter.

Table I

Demographics and Clinical Information for Subjects with and without post-TBI depression.

\begin{tabular}{|c|c|c|c|}
\hline Variable & $\begin{array}{c}\text { Controls } \\
(\mathrm{N}=5)\end{array}$ & $\begin{array}{r}\text { Post-TBI I } \\
(\mathrm{N}=\end{array}$ & $\begin{array}{l}\text { Depression } \\
=5)\end{array}$ \\
\hline Age & $44.4(12.52)$ & 46.4 & $(15.24)$ \\
\hline$\%$ Female & $4 \quad(80 \%)$ & 1 & $(20 \%)$ \\
\hline Months since TBI (mean, sd) & $\mathrm{n} / \mathrm{a}$ & 26 & $(22)$ \\
\hline Montgomery Aspburgh Depression Scale & $\mathrm{n} / \mathrm{a}$ & 15.29 & $(11.3)$ \\
\hline Measure of TBI severity & $\mathrm{n} / \mathrm{a}$ & & 3 mild; 2 severe $^{+}$ \\
\hline
\end{tabular}

${ }^{+}$Determined by Loss of Consciousness (<30 mins, Mild, $>24$ hours, Severe). Data are presented as means (standard deviations).

Table II

Comparison of TBI Depressed and Control Subjects on MRS Findings.

\begin{tabular}{|c|c|c|c|c|c|}
\hline Area & Ratio & $\begin{array}{c}\text { Depressed } \\
\text { TBI }(n=5)\end{array}$ & $\begin{array}{l}\text { Controls } \\
(\mathrm{n}=5)\end{array}$ & $\mathrm{t}, \mathrm{p}$ & $\begin{array}{c}\text { Effect } \\
\text { Size }\end{array}$ \\
\hline Frontal White Matter & $\begin{array}{l}\text { NAA/Cho } \\
\text { NAA/Cr } \\
\text { Cho/Cr }\end{array}$ & $\begin{array}{l}1.62(.43) \\
1.95(.56) \\
1.30(.38)\end{array}$ & $\begin{array}{l}1.57(.25) \\
3.10(1.08) \\
1.89(.99)\end{array}$ & $\begin{array}{l}\mathrm{t}(8)=1.91, \mathrm{p}=0.853 \\
\mathrm{t}(8)=-2.12, \mathrm{p}=.067 \\
\mathrm{t}(8)=1.235, \mathrm{p}=0.252\end{array}$ & $\begin{array}{r}.16 \\
-1.49 \\
-.87\end{array}$ \\
\hline Frontal Gray Matter & $\begin{array}{l}\text { NAA/Cho } \\
\text { NAA/Cr } \\
\text { Cho/Cr }\end{array}$ & $\begin{array}{l}1.65(.25) \\
1.68(.70) \\
1.39(.39)\end{array}$ & $\begin{array}{l}2.40(.43) \\
2.69(1.05) \\
1.06(.21)\end{array}$ & $\begin{array}{l}\mathbf{t}(\mathbf{8})=\mathbf{- 3 . 4 0}, \mathbf{p}=\mathbf{0 . 0 0 9} \\
\mathrm{t}(8)=-1.78, p=0.113 \\
\mathrm{t}(8)=-1.681, p=0.13\end{array}$ & $\begin{array}{r}-2.38 \\
-1.27 \\
1.19\end{array}$ \\
\hline Basal Ganglia & $\begin{array}{l}\text { NAA/Cho } \\
\text { NAA/Cr } \\
\text { Cho/Cr }\end{array}$ & $\begin{array}{l}1.43(.35) \\
1.73(.28) \\
1.21(.36)\end{array}$ & $\begin{array}{l}1.45(.29) \\
2.46(.65) \\
1.54(.62)\end{array}$ & $\begin{array}{l}t(8)=-.107, p=0.918 \\
\mathbf{t}(8)=\mathbf{- 2 . 3 0} \quad \mathbf{p}=\mathbf{0 . 0 5 0} \\
\mathrm{t}(8)=1.052, p=0.32\end{array}$ & $\begin{array}{r}-.07 \\
-1.63 \\
-.74\end{array}$ \\
\hline Thalamus & $\begin{array}{l}\text { NAA/Cho } \\
\text { NAA/Cr } \\
\text { Cho/Cr }\end{array}$ & $\begin{array}{l}1.32(.19) \\
1.88(.17) \\
1.57(.17)\end{array}$ & $\begin{array}{l}1.61(.20) \\
2.04(.75) \\
1.32(.29)\end{array}$ & $\begin{array}{l}\mathbf{t}(\mathbf{8})=\mathbf{- 2 . 3 2}, \mathbf{p}=\mathbf{0 . 0 4 9} \\
\mathrm{t}(8)=-.459, \mathrm{p}=0.658 \\
\mathrm{t}(8)=-1.64, \mathrm{p}=0.139\end{array}$ & $\begin{array}{r}-1.66 \\
-.33 \\
1.17\end{array}$ \\
\hline
\end{tabular}

Data are presented as means (standard deviations). Statistical significance is indicated by bold interface. Effect size was calculated by subtracting the means of each group, divided by the pooled standard deviation. 
There was no significant difference between the TBI depressed subjects and the normal controls in the $\mathrm{Cho} / \mathrm{Cr}$ ratio in any of these regions.

The MRI findings of the five TBI depressed subjects are shown in Table III.

\section{Discussion}

Subjects with post-TBI depression (Cases) exhibited decreased NAA/Cho or $\mathrm{NAA} / \mathrm{Cr}$ ratios in the frontal GM, basal ganglia, and thalamus, compared to subjects lacking a history of TBI or depression (Controls). The reduced NAA/Cho ratio could be the result of reduced NAA or increased choline. If both are reduced, the ratio will be unchanged. Similarly, the reduced NAA/Cr could be the result of reduced NAA or increased creatine. If both are reduced, the ratio will be unchanged. However, since there was no significant difference in the $\mathrm{Cho} / \mathrm{Cr}$ ratio between the two groups, it is very likely that the reduced NAA/Cho and NAA/Cr ratios are due to reduced NAA levels. These results further suggest that the reduced NAA among cases is probably due to neuronal-axonal damage. Given that our control subjects lack both a history of TBI and depression, the reduction in NAA in post-TBI depression may be due to : brain injury from TBI (hypothesis1) or the presence of depressed mood (hypothesis 2).

Table III

FLAIR MRI findings in Cases and Control.

CASES (TBI Depressed)

\#1

\#2

\# 3

\#4

\# 5
Damage to right temporal lobe, right frontal lobe and posterior left frontal lobe. Hyperintense signal intensities in the periventricular areas and corpus callosum. Surgery right frontal-parietal calvarium

Punctate foci of increased signal in the frontal lobe with small area of encephalomalacia in the right posterior-inferior frontal lobe

Small area of encephalomalacia in the left temporal lobe. Mildly enlarged ventricles

Subcentimter are of signal intensity in the right frontal region, possibly due to meningioma. Punctate subcortical high signal intensity foci in the left frontal region

Extensive bifrontal hemorrhagic brain injury with encephalomalacia and gliosis, left worse than right. Punctate areas of white matter damage in the subcortical regions
Hypothesis 1 is supported by our findings that four out of five post-TBI depression cases had anatomical injury to the frontal lobes on MRI, and two out of five subjects injuries to the subcortical regions (Table III). Other researchers have also shown that TBI is associated with reduced NAA/Cho ratios in several brain regions. Yoon et al. (2005) using image-guided localized in vivo ${ }^{1} \mathrm{H}$ MRS have shown decreased NAA /Cr ratio and increased $\mathrm{Cho} / \mathrm{Cr}$ and myoinositol/Cr ratios in the parietal white matter (WM) in the TBI subjects compared to normal controls . No difference in these ratios were seen in the occipital GM, suggesting that WM tracts are particularly vulnerable to damage from TBI. Other studies have also shown decreased NAA, both in areas of obvious injury and in normal appearing GM and WM (Condon et al. 1998, Garnett et al. 2001). 
Thus, reduced NAA/Cho and NAA/Cr ratios may be markers of diffuse axonal injury seen secondary to TBI. However, the anatomic distribution of our findings (frontal GM, basal ganglia, and thalamus) are quite different from these WM abnormalities.

Hypothesis 2, that depression is associated with the observed reduction in NAA, is indirectly supported by the anatomic distribution of MRS abnormalities in major depressive disorder (MDD). Vythilingam et al. (2004) compared subjects with MDD and normal controls using automated mutivoxel chemical-shift imaging proton MRS techniques. MDD patients were found to have a significantly lower mean NAA/Cr ratio in the caudate and higher $\mathrm{Cho} / \mathrm{Cr}$ ratio in the putamen. No significant difference between the two groups were found in whole brain measurements. These results suggest basal ganglia abnormalities in subjects with major depression. Other MRS studies have shown reduced myoinositol levels in the frontal regions in depressed subjects compared to controls (Coupland 2005) and decreased glutamate/glutamine levels in the anterior cingulate region (Auer et al. 2000). Myoinositol and glutamate levels were not measured in the present study. Studies using functional imaging techniques have shown abnormalities in the frontalsubcortical tracts in depressed subjects compared to healthy controls. In a review article on positron emission tomography (PET) studies of major depression, Videbech (2000) has concluded that depression is associated with abnormalities in the frontal lobes, limbic system and basal ganglia.

The anatomic distribution of our results is also similar to imaging studies of depression in other neurologic diseases. These have been summarized by Mayberg (1994) who postulated that depression is associated with bilateral hypometabolism of the orbital inferior prefrontal cortex and temporal cortex, due to disruption of the paralimbic pathways linking frontal cortex, temporal cortex and striatum. Studies using neuroimaging techniques other than MRS have shown metabolic abnormalities in the frontal-basal-ganglia-thalamic circuit in brain injured depressed subjects. In a pilot study comparing the findings of SPECT and neuropsychological testing 5-48 months after a mild TBI in four depressed patients, Umile et al. (1998) found abnormal regional cerebral blood flow in the frontal and temporal regions as well as hemispheric asymmetry (left $>$ right). Mayberg et al. (1990) compared cerebral glucose metabolism using PET in Parkinson's disease subjects with and without depression and agematched healthy subjects. Overall, these findings in MDD and Parkinson's disease are anatomically similar (though not identical) to our findings, and suggest that the NAA reductions we observed are due to depressed mood rather than TBI per se (supporting hypothesis 2).

The major strength of this study is that this is the first report on MRSI findings in subjects with post-TBI depression. The major limitations are the small size of this pilot study limiting its power to generate statistically significant findings, and the control group which differs from post-TBI depression in two conditions (lack of depression and lack of TBI). Thus, these findings must be considered only preliminary; in future studies must be designed to include a group of post-TBI subjects without depression and subjects with primary depression. The absence of differences in the NAA/Cho or NAA/Cr ratios in the frontal white matter among cases and controls in this study, may probably be due to the small number of subjects in the study. 
Another limitation is that we have only included TBI subjects who meet criteria for major depressive disorder. It is becoming increasingly clear that the spectrum of affective disorder in neurological illness may have different symptom clusters and natural history than major depressive disorder, and that new and broader definitions of depression may be useful in defining phenotypes for clinical and biological studies (Rosenberg et al. 2005, Lyketsos et al. 2001). In addition, we limited our studies to a small number of regions of interest (ROI), and given the implication of WM injury in TBI other regions of the brain susceptible to diffuse axonal injury such as splenium of the corpus callosum, internal capsule and mid-brain, should also be analyzed in future studies.

\section{Conclusion}

Major depression is a debilitating psychiatric sequela of TBI. We present the first MRS findings from a pilot study in post-TBI depression. We have detected reduced NAA in frontal GM, basal ganglia and thalamus in the TBI depressed group compared to nonTBI non-depressed controls. These results suggest that MRS is a promising modality for development of biomarkers of disease (and, conceivably treatment response) in mood disorders. However, larger studies with better control groups are needed to further substantiate these findings.

\section{References}

Auer DP, Putz B, Kraft E, Lipinski B, Schill J, Holsboer F. Reduced glutamate in the anterior cingulate cortex in depression: an in vivo proton magnetic resonance spectroscopy study. Biol Psychiatry 2000; 47(4): 305-313.

Bachelard H, Badar-Goffer R.NMR spectroscopy in neurochemistry. Review. J Neurochem 1993; 61(2): 412429.

Brooks WM, Friedman S, Gasparovic C: Magnetic resonance spectroscopy in TBI. J Head Trauma Rehabil 2001; 16 (2): 149-164.

Condon B, Oluoch-Olunya D, Hadley D, Teasdale G, Wagstaff A. J Early $1 \mathrm{H}$ magnetic resonance spectroscopy of acute head injury: four cases. Neurotrauma 1998; 15(8): 563-571.

Coupland NJ, Ogilvie CJ, Hegadoren KM, Seres P, Hanstock CC, Allen PS. Decreased prefrontal Myo-inositol in major depressive disorder. Biol Psychiatry 2005; 57(12): 1526-1534.

Deb S, Lyons I, Koutzoukis C, Ali I, McCarthy G. Rate of psychiatric illness 1 year after traumatic brain injury. Am J Psychiatry 1999; 156(3): 374-378.

Duyn JH, Gillen J, Sobering G, van Zijl PCM, Moonen CT. Multisection proton MR spectroscopic imaging of the brain. Radiology 1993; 188: 277-282.

Garnett MR, Blamire AM, Corkill RG, Cadoux-Hudson TA, Rajagopalan B, Styles P. Early proton magnetic resonance spectroscopy in normal-appearing brain correlates with outcome in patients following traumatic brain injury. Brain 2000; 123: 2046-2054.

Hibbard MR, Uysal S, Kepler K, Bogdany J, Silver J. Axis I psychopathology in individuals with traumatic brain injury. J Head Trauma Rehabil 1998; 13(4): 24-39.

Jorge R, Robinson R, Arndt S et al.: Comparison between acute and delayed onset depression following traumatic brain injury. J Neuropsychiatry ClinNeurosci 1993; 5: 43-49.

Jorge RE, Robinson RG, Starkstein SE, Arndt SV. Influence of major depression on 1-year outcome in patients with traumatic brain injury. J Neurosurg 1994; 81: 726733.

Jorge RE, Robinson RG, Moser D, Tateno A, CrespoFacorro B, Arndt S. Major depression following traumatic brain injury. Arch Gen Psychiatry 2004; 61(1): 42-50.

Kumar A, Thoms A, Layretsky J et al:: Frontal white matter biochemical abnormalities in late-life major depression detected with proton magnetic resonance spectroscopy. Am J Psychiatry 2002; 159(4): 630-636.

Lyketsos CG, Sheppard JM, Steinberg M, Tschanz JA, Norton MC, Steffens DC, Breitner JC. Neuropsychiatric disturbance in Alzheimer's disease clusters into three groups: the Cache County study. Int J Geriatr Psychiatry 2001; 16: 1043-1053. 
Lyoo IK and Renshaw PF: Magnetic Resonance Spectroscopy: Current and future applications in psychiatric research. Biol Psychiatry 2002; 51: 195-207.

Mayberg HS, Starkstein SE, Sadzot B, Preziosi T, Andrezejewski PL, Dannals RF, Wagner HN Jr, Robinson RG: Selective hypometabolism in the inferior frontal lobe in depressed patients with Parkinson's disease.Ann Neurol 1990 ; 28(1): 57-64.

Mayberg HS: Frontal Lobe Dysfunction in secondary depression. J Neuropsychiatry ClinNeurosci 1994; 6: 428442.

McAllister TW. Neuropsychiatric sequelae of head injuries. Psychiatr Clin North Am 1992; 15(2): 395-413.

Moore GJ, Galloway MP: Magnetic resonance spectroscopy: neurochemistry and treatment effects in affective disorders. A Review. Psychopharmacol Bull 2002; 36(2): $5-23$.

Murata, T, Kimura H, Omori M et al:: MRI white matter hyperintensities, (1)H-MR spectroscopy and cognitive function in geriatric depression: a comparison of early- and late-onset cases. Int J Geriatr Psychiatry 2001; 16(12): 1129-1135.

Rosenberg PB, Onyike CU, Katz IR, Porsteinsson AP, Mintzer JE, Schneider LS, Rabins PV, Meinert CL, Martin BK, Lyketsos CG; Depression of Alzheimer's Disease Study. Clinical application of operationalized criteria for 'Depression of Alzheimer's Disease'. Int J Geriatr Psychiatry 2005; 20: 119-127.
Soher BJ, van Zijl PCM, Duyn JH, Barker PB. Quantitative proton MR spectroscopic imaging of the human brain. Magn Reson Med 1996; 35: 356-363.

Umile EM, Plotkin RC, Sandel ME: Functional assessment of mild traumatic brain injury using SPECT and neuropsychological testing. Brain Injury 1998; 7: 577-594.

Videbech P.PET measurements of brain glucose metabolism and blood flow in major depressive disorder: a critical review. Acta Psychiatr Scand 2000; 101(1): 11-20.

Vythilingam M, Charles HC, Tupler LA, Blitchington T, Kelly L, Krishnan KR. Focal and lateralized subcortical abnormalities in unipolar major depressive disorder: an automated multivoxel proton magnetic resonance spectroscopy study. Biol Psychiatry 2003; 54(7): 744-750.

Yoon SJ, Lee JH, Kim ST, Chun MH: Evaluation of traumatic brain injured patients in correlation with functional status by localized 1H-MR spectroscopy. Clin Rehabil 2005; 19 (2) 209-215.

Address for correspondence:

Vani Rao, MD

Division of Geriatric Psychiatry \& Neuropsychiatry

Department of Psychiatry

Johns Hopkins School of Medicine

Baltimore, MD 21287

Email:vrao@jhmi.edu

USA 